\title{
Patient perspectives on barriers to participating in cardiac rehabilitation
}

\author{
Mettananda $\mathbf{C}^{1}$, Ismail $\mathbf{I}^{2}$, Mettananda $\mathrm{S}^{3}$ \\ Journal of the Ceylon College of Physicians, 2020, 51, 91-95
}

\begin{abstract}
Despite proven benefits, attendance at cardiac rehabilitation (CR) is poor worldwide. We aimed to describe patient perspectives on barriers to attend CR in Sri Lanka.
\end{abstract}

A cross-sectional study was conducted at cardiothoracic outpatient clinics of the National Hospital of Sri Lanka from May to July 2019. Post-cardiac surgery patients who were referred to $C R$ but not attending them were recruited.

80 patients (male-51.3\%, mean age $59.5 \pm 11.7$ years) who were not attending CR were studied. $60 \%$ had to travel more than $50 \mathrm{~km}$ to attend CR. Main reasons for non-attendance were transportation difficulties (36\%), non-availability of nearby CR centres (36\%), unaware of being referred to $C R$ due to communication lapses (35\%) and lack of awareness of the importance of CR (31\%).

Therefore, providing clear information on cardiac rehabilitation, incorporating patient education programmes in cardiac surgery protocols and establishing new cardiac rehabilitation centres are recommended.

Key words: cardiac rehabilitation, patient perspectives, barriers, Sri Lanka

\section{Introduction}

Cardiac rehabilitation ( $C R$ ) is a medically supervised, structured programme designed to improve cardiovascular health following coronary heart disease (CHD) and cardiac surgeries. This includes health education on cardiovascular risk reduction, physical activity and stress management. ${ }^{1-3} \mathrm{CR}$ has proven beneficial effects on reducing cardiovascular mortality and morbidity, and improvement in risk factor control and quality of life. ${ }^{4,5}$ It is also reported to be beneficial in reducing reinfarction rate and all-cause mortality following myocardial infarction, percutaneous coronary intervention, coronary artery bypass graft and chronic heart failure..$^{6-9}$

Despite evidence on the beneficial effects, patient attendance at CR is poor which varies from $20-50 \%$ in different countries. ${ }^{1,7,10,11,12}$ Data on uptake of CR among South Asians is limited and the available data reports very poor uptake. ${ }^{13}$ There is no data on the uptake of CR among patients in Sri Lanka which anecdotally is said to be poor. In this study we aim to describe the patient perspectives on barriers to participate in $\mathrm{CR}$ programmes of the National Hospital of Sri Lanka.

\section{Methods}

A cross-sectional study was conducted at the adult cardiothoracic outpatient clinic of the National Hospital of Sri Lanka which has the largest CR programme of the country. The study was conducted from May to July 2019. All patients attending outpatient cardiothoracic clinic following cardiac surgery were screened to identify patients who do not attend CR programmes despite being referred to. This was done by perusing post-surgical clinic notes. CR programme consists of 2 sessions a week for 4 weeks. Patients

${ }^{1}$ Department of Pharmacology, Faculty of Medicine, University of Kelaniya, Ragama, Sri Lanka, ${ }^{2}$ Cardiac RehabilitationI Unit, National Hospital of Sri Lanka, Colombo, Sri Lanka, ${ }^{3}$ Department of Paediatrics, Faculty of Medicine, University of Kelaniya, Ragama, Sri Lanka.

Correspondence: CM, e-mail: Chamila@kln.ac.lk, chamila@hotmail.com

(iD) https://orcid.org/0000-0002-3328-1553

Received 17 July 2020, accepted 20 September 2020.

This is an open-access article distributed under the terms of the Creative Commons Attribution License, which permits unrestricted use, distribution, and reproduction in any medium, provided the original author and source are credited. 
who have never attended CR programmes or had addended less than 2 sessions of the 8 sessions were defined as CR non-attendees. All CR non-attendees were recruited into the study after obtaining informed written consent. Data on reasons for non-attendance were collected using an interviewer-administered questionnaire by interviewing study participants. Data were analysed using SPSS statistics version 22.0. Continuous variables were reported as means with standard deviation (SD), and categorical variables were reported as percentages. The significance level was set at $p<0.05$.

Ethics approval was obtained from the Ethics Review Committee of the Faculty of Medicine, University of Kelaniya, Sri Lanka. Informed written consent of all the patients were obtained.

\section{Results}

A total of 80 patients who were not attending CR clinics (male $51.3 \%$, mean age $59.5 \pm 11.7$ years, age range 29-78 years) were recruited. Their baseline characteristics are shown in Table 1. A majority [58 $(72.5 \%)]$ of patients had undergone coronary artery bypass grafting while $7(8.8 \%)$ had cardiac valve repairs and another 15 (18.8\%) had surgery for congenital heart diseases. Of the CR non-attendees, $60 \%$ had studied beyond Grade 10, 53\% had a monthly income equal or above Rs. 30,000, 52\% were employed and 50\% had a chronic or acute medical problem necessitating them to be on medications. $92.7 \%$ had to travel more than $25 \mathrm{~km}$ to attend $\mathrm{CR}$ programmes.

Table 2 shows the reasons for CR programme non-attendance. Of the CR non-attendees, 28 (35.0\%) were unaware of them being referred to $C R$. The other three main reasons were transportation difficulties due to long-distance in 29 (36.3\%), lack of nearby CR centres in 29 (36.3\%) and lack of awareness about the importance of CR in 25 (31.3\%). However, coexistent medical problems and lack of commitment were not the problems for non-attendance in many (Table 2).

Table 1. Baseline characteristics $(n=80)$

\begin{tabular}{ll}
\hline Characteristics & Number (\%) \\
Male $\mathrm{n}(\%)$ & $41(51.2)$ \\
Age ( years) mean (SD) & $59.5 \pm 11.7$ \\
Civil status; married $\mathrm{n}(\%)$ & $70(87.5)$ \\
Level of education, $\mathbf{n}$ (\%) & \\
Up to grade 1-5 & $5(6.3 \%)$ \\
Up to grade 6-10 & $27(33.8 \%)$ \\
Completed O/L & $19(23.8 \%)$ \\
Completed A/L & $14(17.5 \%)$ \\
Graduates & $15(18.8 \%)$ \\
Ethnicity & \\
Sinhalese & $60(75.0)$ \\
Tamils & $14(8.8)$ \\
Muslims & $6(7.5)$ \\
Income, $\mathbf{n}$ (\%) & \\
Rs. $10000.00-19999.00$ & $19(23.8)$ \\
Rs. 20000.00 - 29999.00 & $18(22.5)$ \\
Rs. $30000.00-39999.00$ & $23(28.7)$ \\
Rs. $40000.00-49999.00$ & $16(20.00$ \\
$>$ Rs. 50000.00 & $4(5.0)$
\end{tabular}




\section{Employment, $\mathbf{n}(\%)$}

Self-employed

Government workers

9 (11.3)

Private sector

$16(20.0)$

Retired

$14(17.5)$

Unemployed

$24(30.0)$

Distance between residence to the CR programme $(\mathrm{km})$

$\begin{array}{lc}\text { Less than } 10 \mathrm{~km} & 2(2.5) \\ 10-25 \mathrm{~km} & 3(3.8) \\ 25-50 \mathrm{~km} & 27(33.8) \\ 50-100 \mathrm{~km} & 18(22.5) \\ \text { More than } 100 \mathrm{~km} & 30(37.5)\end{array}$

Co-morbid medical conditions, $\mathbf{n}(\%)$

Diabetes mellitus

Arthritis on treatment

$6(7.5)$

Chronic lung disease

$11(13.8)$

Chronic kidney disease

$2(2.5)$

Cancer

$1(1.3)$

No other medical problems

$40(50.0)$

Table 2. Reasons for non-attendance at CR programs

\begin{tabular}{ll}
\hline Reasons for not attending CR & Number (\%) \\
\hline Transportation difficulties & $29(36.3)$ \\
Non-availability of nearby CR Centres & $29(36.3)$ \\
Unaware of being referred to CR & $28(35.0)$ \\
Lack of awareness on CR & $25(31.3)$ \\
No proper guidance is given & 20 \\
Thought need to attend only once & 3 \\
Thought medications alone would be adequate & 2 \\
Other co-morbidities & $9(11.3)$ \\
Work responsibilities & $5(6.3)$ \\
Lack of a guardian to accompany & $4(5.0)$ \\
Have to spend a long time as there are a lot of patients & $4(5.0)$ \\
Lack of individual attention & $2(2.5)$ \\
Difficulty in attending on weekdays & $2(2.5)$ \\
nadequate number of available staff on some days & $1(1.3)$ \\
Do not have energy & $11.3)$ \\
& \\
\hline
\end{tabular}




\section{Discussion}

Sri Lanka is a South Asian country with a population of 22 million that experience over 66,000 ischemic heart disease episodes every year. CR was formally established in the country in 2002. At the time of study, there were five CR centres; National Hospital of Sri Lanka, Teaching Hospital Kandy, Teaching Hospital Karapitiya, Teaching Hospital Rathnapura, and Provincial General Hospital Kurunegala, of which could cater for a median of 420 patients per year. ${ }^{14}$ (Figure 1). At present there are four new CR centres; Provincial General Hospital Badulla, Teaching Hospital Peradeniya, Teaching Hospital Kalubowila and Base Hospital Awissawella. The CR centre of the National Hospital of Sri Lanka where this study was conducted is the largest CR centre of the country.

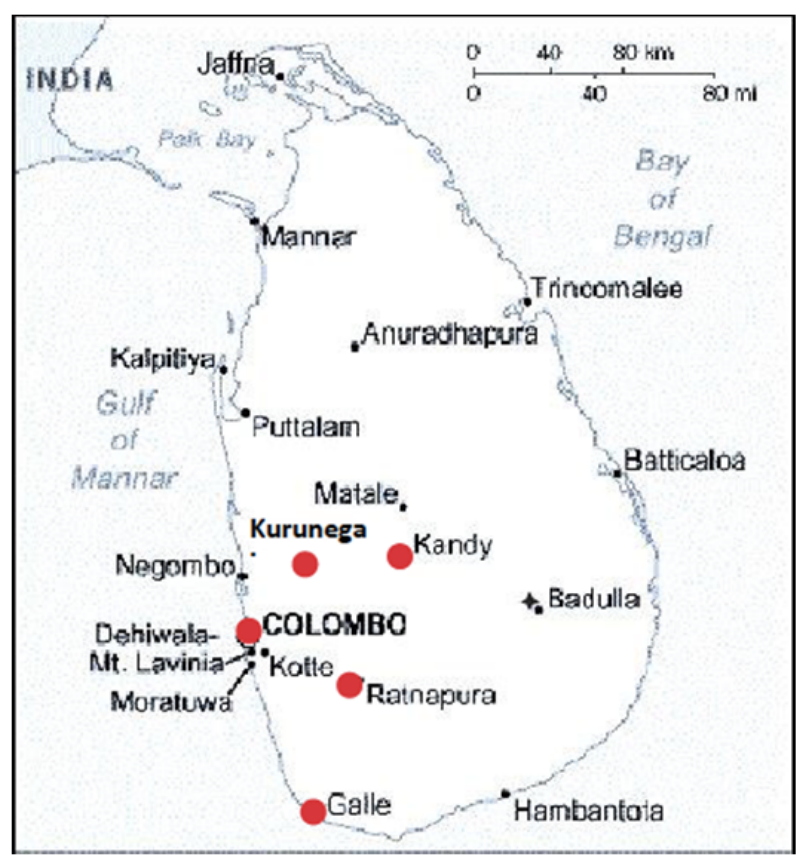

Figure 1. The distribution of cardiac rehabilitation centers in Sri Lanka.

We identified several reasons for poor attendance of Sri Lankan patients to CR programmes. One important factor revealed was lack of easily accessible $\mathrm{CR}$ programmes. More than half of the patients in the study had to travel over $50 \mathrm{~km}$ for their CR centre. This is demanding considering the fact that these patients had undergone major cardiac surgeries and traveling long distances is not easy. Inadequate availability of $\mathrm{CR}$ centres is not a problem unique to Sri Lanka but common to many developing countries in the world. In fact, CR centres are only available in 111 (54\%) countries globally. ${ }^{14}$ Availability ranges from $80 \%$ in the Europe to $17 \%$ in Africa. ${ }^{14}$ The availability of CR programs was shown to be low (24\%) even in large medical centres in China. ${ }^{15}$

Even in the countries where CR facilities are available, under-utilisation is reported. Previous studies have shown that one primary reason for poor uptake of $\mathrm{CR}$ in developed counties was physicians' inertia in referring patients to the available centres. A study done in the USA uncovered that nearly $80 \%$ of patients with indications for CR according to American Heart Association guidelines were not referred for CR. ${ }^{10}$ Elderly, women, patients from ethnic minorities and that of lower socioeconomic communities had significantly low referral rates. ${ }^{16}$ The main reasons behind inadequate referral by physicians were; lack of a proper referral pathway, poor communication between patients and CR teams, lack of knowledge about CR among treating physicians and limited availability of the facility. ${ }^{10,16-18}$ The situation is not different in Europe ${ }^{12}$ and elsewhere. ${ }^{14}$ Poor communication between the physician and the patient at discharge from hospital and lack of awareness among patients of CR were identified as a key factor in our study as well.

Of the appropriately refereed patients, only a minority were attending CR programmes even in developed countries. In a US study among patients who were referred for CR following myocardial infarctions, only $29 \%$ and $48 \%$ were attending CR programmes within 1 and 6 months postoperatively respectively. ${ }^{19}$ Several reasons were identified as causes for this. The reasons were; problems in accessing the facilities due to transport issues, geographical location, phycological issues, other comorbidities, lack of knowledge on the benefits of CR and pure patient inertia. ${ }^{1,10,19}$ In Sri Lanka, the main reasons we identified for poor patient adherence were transportation difficulties, non-availability of centres close to homes, lack of awareness of being referred to $\mathrm{CR}$ and poor knowledge on CR. However, the presence of co-morbid medical conditions or patient inertia were not major barriers to participation in CR of our study cohort.

\section{Conclusions}

This very first study on patient perspectives on poor adherence to CR programmes in Sri Lanka revealed, limited availability of CR programs within easy access, unawareness of being referred to CR secondary to poor communication between physicians and patients on discharge, and poor awareness of patients on the importance of $\mathrm{CR}$, as the main reasons for poor uptake of CR. Therefore, educating patients who underwent cardiac surgeries, providing clear and concise information on the benefits of $\mathrm{CR}$ and the 
process of CR, by physicians or trained nurses on discharge from hospitals is vital. Similarly, incorporating patient education programmes in the cardiac surgery protocols and establishing new cardiac rehabilitation centres in every province are important to increase the use of CR in Sri Lanka.

\section{Conflicts of interest}

None.

\section{References}

1. Dalal HM, Doherty P, Taylor RS. Cardiac rehabilitation. BMJ 2015; 351: h5000.

2. Balady GJ, Fletcher BJ, Froelicher ES, et al. Cardiac rehabilitation programs. A statement for healthcare professionals from the American Heart Association. Circulation 1994; 90(3): 1602-10.

3. Balady GJ, Ades PA, Comoss P, Limacher M, Pina IL, Southard D, et al. Core Components of Cardiac Rehabilitation/ Secondary Prevention Programs. Circulation 2000; 102(9): 1069-73.

4. Sandesara PB, Lambert CT, Gordon NF, et al. Cardiac Rehabilitation and Risk Reduction: Time to "Rebrand and Reinvigorate". J Am Coll Cardiol. 2015; 65(4): 389-95.

5. Anderson L, Oldridge N, Thompson DR, et al. ExerciseBased Cardiac Rehabilitation for Coronary Heart Disease: Cochrane Systematic Review and Meta-Analysis. J Am Coll Cardiol. 2016; 67(1): 1-12.

6. Lawler PR, Filion KB, Eisenberg MJ. Efficacy of exercisebased cardiac rehabilitation post-myocardial infarction: $A$ systematic review and meta-analysis of randomized controlled trials. Am Heart J. 2011; 162(4): 571-84.

7. Rengo JL, Savage PD, Barrett T, Ades PA. Cardiac Rehabilitation Participation Rates and Outcomes for Patients With Heart Failure. J Cardiopulm Rehabil Prev. 2018; 38(1): 38-42.

8. Goel K, Lennon RJ, Tilbury RT, Squires RW, Thomas RJ. Impact of Cardiac Rehabilitation on Mortality and Cardiovascular Events After Percutaneous Coronary
Intervention in the Community. Circulation 2011; 123(21): 2344-52.

9. Pack QR, Goel K, Lahr BD, Greason KL, Squires RW, LopezJimenez F, et al. Participation in Cardiac Rehabilitation and Survival After Coronary Artery Bypass Graft Surgery. Circulation 2013; 128(6): 590-7.

10. MenezesAR, Lavie CJ, Milani RV, Forman DE, King M, Williams MA. Cardiac rehabilitation in the United States. Prog Cardiovasc Dis. 2014; 56(5): 522-9.

11. Jelinek MV, Thompson DR, Ski C, Bunker S, Vale MJ. 40 years of cardiac rehabilitation and secondary prevention in post-cardiac ischaemic patients. Are we still in the wilderness? Int J Cardiol. 2015; 179: 153-9.

12. Humphrey R, Guazzi M, Niebauer J. Cardiac Rehabilitation in Europe. Progress in Cardiovascular Diseases 2014; 56(5): 551-6.

13. Galdas PM, Ratner PA, Oliffe JL. A narrative review of South Asian patients' experiences of cardiac rehabilitation. J Clin Nurs. 2012; 21(1-2): 149-59.

14. Turk-Adawi K, Supervia M, Lopez-Jimenez F, Pesah E, Ding R, Britto RR, et al. Cardiac Rehabilitation Availability and Density around the Globe. E Clinical Medicine. 2019; 13: 31-45.

15. Zhang Z, Pack Q, Squires RW, Lopez-Jimenez F, Yu L, Thomas RJ. Availability and characteristics of cardiac rehabilitation programmes in China. Heart Asia 2016; 8(2): 9.

16. Leon AS, Franklin BA, Costa F, et al. Cardiac Rehabilitation and Secondary Prevention of Coronary Heart Disease. Circulation 2005; 111(3): 369-76.

17. Wenger NK. Current Status of Cardiac Rehabilitation. J Am Coll Cardiol. 2008; 51(17): 1619-31.

18. Arena R, Williams M, Forman DE, et al. Increasing Referral and Participation Rates to Outpatient Cardiac Rehabilitation: The Valuable Role of Healthcare Professionals in the Inpatient and Home Health Settings. Circulation 2012; 125(10): 1321-9.

19. Parashar S, Spertus JA, Tang F, et al. Predictors of early and late enrollment in cardiac rehabilitation, among those referred, after acute myocardial infarction. Circulation 2012; 126(13): 1587-95. 